\title{
Equivalence of String Classical and Quantum Energy beside Equivalence of Wave Packet and Relativistic Velocity in Eucleadian and Curved Space
}

\author{
Mashair Ahmed Mohammed Yousif ${ }^{1}$, Abeer Mohammed Khairy Ahmed ${ }^{2}$, \\ Zainab Mustapha Kurawa ${ }^{3}$, Omer A. M. Elnor ${ }^{4}$, Mubarak Dirar Abd-Alla Yagoub ${ }^{5}$, \\ Ibrahim Mohammed Elfaki El-Tahir 6 , Mohammed Idriss Ahmed ${ }^{5}$, \\ Zoalnoon Ahmed Abeid Allah Saad ${ }^{7}$
}

\footnotetext{
${ }^{1}$ Department of Physics, Faculty of Science and Education at Alkurma, Taif University, Alkhurma, Kingdom of Saudi Arabia; ${ }^{2}$ Department of Physics, Faculty of Art and Science at Al Muznab, Al Qassim University, Al Muznab, Kingdom of Saudi Arabia; ${ }^{3}$ Department of Physics, School of Science Education, Saadatu Rimi College of Education, Kumbotso, Nigeria; ${ }^{4}$ Department of Physics, Faculty of Science, University of Bakhtalruda, Ed Dueim, Sudan; ${ }^{5}$ Department of Physics, Faculty of Science, Sudan University of Science and Technology, Khartoum, Sudan; ${ }^{6}$ Department of Natural and Applied Sciences, College of Science and Humanities at Afif, Sharga University, Afif, Kingdom of Saudi Arabia; ${ }^{7}$ College of Arts and Sciences, King Khalid University, Dhahran, Saudi Arabia
}

Correspondence to: Mashair Ahmed Mohammed Yousif, mubarakdirar@gmail.com Keywords: Plank Energy, Classical Energy, String Quanta, Photons, Electromagnetic Waves, Curved Space, Group Velocity, Wave Packet

Received: May 17, 2020

Accepted: July 20, 2020

Published: July 23, 2020

Copyright (c) 2020 by author(s) and Scientific Research Publishing Inc.

This work is licensed under the Creative Commons Attribution International License (CC BY 4.0).

http://creativecommons.org/licenses/by/4.0/

(c) (i) Open Access

\section{ABSTRACT}

Plank quantum and classical string energy relations seem to be uncorrelated. This work correlated them. The relativistic energy-momentum relation has been used together with plank and de Brogglie hypothesis to prove that the wave group velocity is equal to the particle velocity in both ordinary and curved space. The Plank energy relation is shown also to be related to the classical energy relation of an oscillating string. Starting from plank energy relation for $\mathbf{n}$ photons and performing integration, the expression of classical string energy was obtained. This means that one can treat electromagnetic waves as a collection of continuous photons having frequencies ranging from zero to w. Conversely, starting from classical string energy relation by differentiating it with respect to angular frequency, the plank quantum energy for $\mathrm{n}$ photons has been found. This means that the quanta results from separation of the electromagnetic waves to single isolated waves. Each wave consists of $n$ photons or quanta. 


\section{INTRODUCTION}

Atoms were first considered a small spherical bodies consisting of electrons, protons and neutrons. The electrons, protons and neutrons were also treated as very tiny small spheres. This version was accepted for a long time $[1,2]$. Unfortunately, the development of elementary particle and unification theories encourages and forces scientists to try to change this version so as to surpass some difficulties associated with this view. This includes the infinite self mass and the problem of neutrino mass $[3,4]$. The neutrino mass problem stems from the fact that the solar neutrino mass for neutrinos that comes from the sun is different from some species reaching the earth [4].

Another approach concerning the nature of elementary particles was proposed by De Brogglie. He suggested that these particles behave as matter wave packets. This hypothesis was verified experimentally by observing electron diffraction when reflected by a crystal [5].

Mathematically, it was also proved that wave packet group velocity is equal to the particle velocity using special relativistic mass energy relation [6].

The failure of spherical models of elementary particles, in addition to the success of De Brogglie matter wave hypothesis encourages some scientists to propose the so-called string theory $[7,8]$. There are many models based on string theory, but they all suggest that elementary particles behave as vibrating strings [9]. Though the beauty of this theory has no wide results and evidences that can explain most of elementary particle phenomena.

However, recently some string models proposed by M. Dirar and others succeeded in constructing simple mathematics and few hypotheses to solve some of these problems [10-12]. In this work, string model is used again to reconcile quantum and classical relations for energy and speed. This is done in section (2). Section (3) is devoted for conclusion.

\section{String energy and velocity according to classical and quantum laws}

According to Plank and De Broggile hypothesis, the energy $E$ and momentum $P$ of each quantum having frequency and wavelength $\lambda$ are given by:

$$
E=h f=\hbar w p=h / \lambda=\hbar k
$$

The De Brogglie hypothesis states that atomic particles can be treated as a wave group (wave packet) formed from the interference of waves having different wave lengths, $\lambda$, and different frequencies $f$. The velocity of it is given by:

$$
\begin{gathered}
v_{g}=\frac{\mathrm{d} W g}{\mathrm{~d} K g}=\frac{\mathrm{d} \hbar w g}{\mathrm{~d} \hbar k g}=\frac{\mathrm{d} E}{\mathrm{~d} P} \\
\hbar=h / 2 \pi h=\text { plank constant } \\
w=2 \pi f=\text { angular frequency } \\
k=2 \pi / \lambda=\text { wave number }
\end{gathered}
$$

There are two relativistic expressions for $E$

$$
E=\left(C^{2} P^{2}+m_{o}^{2} C^{4}\right)^{\frac{1}{2}}
$$

and

$$
E=m c^{2}=\frac{m_{o} c^{2}}{\beta^{\frac{1}{2}}}=m_{o} c^{2} \beta^{-\frac{1}{2}}
$$

where

$$
\beta=1-v^{2} / c^{2}=1-\frac{m^{2} v^{2} c^{2}}{m^{2} c^{4}}=1-\frac{P^{2} c^{2}}{E^{2}}
$$


Consider now the expression of group velocity (wave packet) using energy momentum relation (3). According to this relation, the group velocity is given by:

$$
v_{g}=\frac{\mathrm{d} E}{\mathrm{~d} P}=\frac{1}{2}\left(c^{2} P^{2}+m_{o}^{2} C^{4}\right)^{-\frac{1}{2}}\left(2 c^{2} P\right)=\frac{C^{2} P}{E}
$$

For zero rest mass, Equation (3) gives

$$
E=C P
$$

Thus Equation (6) gives

$$
v_{g}=\frac{C^{2} P}{C P}=C
$$

which means that any particle which has zero rest mass moves with the speed of light. However, using Equation (4) in Equation (6) gives

$$
v_{g}=\frac{C^{2} P}{m c^{2}}=\frac{p}{m}=\frac{m v}{m}=v
$$

which means that any particle that have no zero rest mass the group velocity is equal to the particle velocity.

Another proof can be made by using Equations (4) and (5) to get

$$
\frac{\mathrm{d} E}{\mathrm{~d} P}=m_{o} c^{2}\left(\frac{1}{2} \beta^{-3 / 2}\right) \frac{\mathrm{d} \beta}{\mathrm{d} P}
$$

With the aid of Equation (5), one gets

$$
\begin{aligned}
\frac{\mathrm{d} E}{\mathrm{~d} P} & =-\frac{1}{2} m_{o} c^{2} \beta^{-\frac{3}{2}} \frac{\mathrm{d} \beta}{\mathrm{d} P} \\
& =-\frac{1}{2} \frac{E}{\beta}\left[-\frac{2 P c^{2}}{E^{2}}+\frac{2 P^{2} c^{2}}{E^{3}} \frac{\mathrm{d} E}{\mathrm{~d} P}\right] \\
& =\frac{1}{\beta}\left[\frac{P c^{2}}{E}-\frac{P^{2} c^{2}}{E^{2}} \frac{\mathrm{d} E}{\mathrm{~d} P}\right]
\end{aligned}
$$

Hence

$$
\begin{gathered}
{\left[1+\frac{P^{2} c^{2}}{\beta E^{2}}\right] \frac{\mathrm{d} E}{\mathrm{~d} P}=\frac{P c^{2}}{\beta E}} \\
\frac{1}{E^{2} \beta}\left[\beta E^{2}+P^{2} C^{2}\right] \frac{\mathrm{d} E}{\mathrm{~d} P}=\frac{P c^{2}}{\beta E} \\
{\left[E^{2}-P^{2} C^{2}+P^{2} C^{2}\right] \frac{\mathrm{d} E}{\mathrm{~d} P}=E P c^{2}} \\
\frac{\mathrm{d} E}{\mathrm{~d} P}=\frac{E P c^{2}}{E^{2}}=\frac{P c^{2}}{E}=\frac{P c^{2}}{m c^{2}}=\frac{P}{m}=\frac{m v}{m}=v
\end{gathered}
$$

Thus according to Equations (2) and (12) the group velocity is given by:

$$
v_{g}=\frac{\mathrm{d} E}{\mathrm{~d} P}=v
$$

Thus the group velocity is equal to the particle velocity. Also Classical and quantum expressions for 
string can be reconciled to each other. To do this consider of photons (or quanta) having continuous frequency ranging from 0 to $f=w / 2 \pi$. Thus their energy is given by:

$$
E=n \int h w \mathrm{~d} w=\frac{n}{2} h w^{2}
$$

where $w$ is the angular frequency. However for classical oscillator the energy of an oscillator takes the form:

$$
E=\frac{1}{2} m A^{2} w^{2}
$$

But the amplitude $A$ is related to the number of photon, since it is proportional to it. The intensity is proportional to both $A^{2}$ and $n$. thus, one can write

$$
n=c o A^{2}
$$

Co is a constant parameter Inserting Equation (16) in (14), and equating (14) and (15) gives

$$
\frac{1}{2} c o A^{2} h w^{2}=\frac{1}{2} m A^{2} w^{2}
$$

Thus the mass of any particle can be related to the plank constant and co according to the relation.

$$
m=c o h
$$

Thus according to Equations (14) and (15) the matter or electromagnetic wave can be considered as resulting from the summation or interaction of photons having continuous frequencies ranging from 0 to $\mathrm{W}$

One can link the classical and quantum expressions for a string by differentiating the classical energy relation in Equation (15) to get the energy of a quanta to be

$$
E_{q}=\frac{\mathrm{d} E}{\mathrm{~d} w}=m A^{2} w
$$

which means that it is the energy per frequency, i.e, the energy of one complete wave. This resemble the Plank quantum energy for $n$ photons, which is given by

$$
E_{q}=n \hbar w
$$

Comparing Equations (19) and (20) gives

$$
m A^{2}=n \hbar
$$

Using relation (16), one gets.

$$
m A^{2}=c o A^{2} h
$$

Again

$$
m=c o h
$$

which means that the energy of one complete discrete isolated wave is equal to the energy of $n$ quanta. This means that one can visualize the matter or electromagnetic waves. Within the frame work of quantum hypothesis as an isolated discrete waves. Each wave consists of $n$ quanta.

Let us find now the parameter co for electron matter waves and electron quanta. According to Equation (23), since the electron mass is

$$
m=9.1 \times 10^{-31} \mathrm{~kg}
$$

Thus

$$
c o=(\mathrm{m} / \hbar)=\frac{9.1 \times 10^{-31}}{6.6 \times 10^{-34}}=\frac{91}{66} \times 10^{3}
$$


One can also test what happens to matter or electromagnetic waves in a curved space. This can be done using the expression of $E$ in a curved space which is given according to the generalized special relativity to be

$$
g_{00} E^{2}=p^{2} c^{2}+m_{o}^{2} c^{4}
$$

Thus the energy is given by

$$
E=g_{00}^{-\frac{1}{2}}\left(p^{2} c^{2}+m_{o}^{2} c^{4}\right)^{\frac{1}{2}}
$$

According to Equation (6) the group velocity takes the form

$$
\begin{aligned}
v_{g} & =g_{00}^{-\frac{1}{2}}\left(p^{2} c^{2}+m_{o}^{2} c^{4}\right)^{-\frac{1}{2}}\left(P C^{2}\right) \\
& =g_{00}^{-1} \frac{P C^{2}}{E}=g_{00}^{-1} \frac{P C^{2}}{m C^{2}}=g_{00}^{-1} \frac{m v}{m}=g_{00}^{-1} v
\end{aligned}
$$

But for curved space and ordinary space

$$
\mathrm{d} s^{2}=c^{2} \mathrm{~d} t_{c}^{2}+\mathrm{d} x_{c}^{2}=c^{2} g_{00} \mathrm{~d} t^{2}+g_{x x} \mathrm{~d} x^{2}
$$

Thus the time $t_{c}$ and displacement $x_{c}$ in a curved space are given by

$$
\mathrm{d} t_{c}=\sqrt{g_{00}} \mathrm{~d} t \mathrm{~d} x_{c}=\sqrt{g_{x x}} \mathrm{~d} x=\left(g_{00}\right)^{\frac{1}{2}} \mathrm{~d} x
$$

When $t$ and $x$ stands for the Eucleadian space, and the schwarzhild solution requires

$$
g_{x x}=g_{00}^{-1}
$$

Thus the velocity $v_{c}$ in a curved space is given by

$$
v_{c}=\frac{\mathrm{d} x_{c}}{\mathrm{~d} t_{c}}=g_{00}^{-1} \frac{\mathrm{d} x}{\mathrm{~d} t}=g_{00}^{-1} v
$$

In view of Equations (27) and (31)

$$
v_{g}=v_{c}
$$

This indicates that even in a curved space the group velocity is equal to the particle velocity.

\section{CONCLUSION}

The equality of wave group and particle speeds has been derived using the energy - momentum relation instead of using relativistic mass and momentum relation. The classical sting oscillator and plank quantum string energy relations have been found to be linked with each other by vowelizing the electromagnetic waves as collection of photons having continuous frequency ranging from zero to $\mathrm{w}$. The quantum has been also shown to be an isolated particles existing inside each separated wave. Extending the De Broggle hypothesis to the curved space shows also that the wave group speed is equal to the particle speed.

\section{CONFLICTS OF INTEREST}

The authors declare no conflicts of interest regarding the publication of this paper.

\section{REFERENCES}

1. Mc Mahon, D. (2009) String Theory. Mc Graw Hill, New York.

2. Sabo, R. (2004) An Introduction to String Theory and D-Brane Dynamics. Imperial College Press, London. https://doi.org/10.1142/p312 
3. Maggiore, M. (2009) A Modern Introduction to Quantum Field Theory. Oxford University Press, New York.

4. Mohammed Saeed, D., Dirar, M., Ibrahim Hassan, H. and Sawsan, A.E. (2018) Generation of Elementary Particles inside Black Holes at Plank Time. International Journal of Innovative Science, Engineering \& Research, 5, 40-47.

5. Schiff, L.I. (1984) Quantum Mechanics. Mc Graw Hill, Tokyo.

6. Derek, F.L. (1982) An Introduction to Tensor Calculus and Relativity. John Wiley and Sons, New York.

7. Zwiebach, B. (2004) A First Course in String Theory. Cambridge University Press, New York. https://doi.org/10.1017/CBO9780511841682

8. Kaku, M. (2000) String Conformal Fields and M-Theory. Springer-Verlag, New York. https://doi.org/10.1007/978-1-4612-0503-6

9. Albuqomi, N.I.A.M.H., Ahmed, I.M., Ahmed, M.I., Khairi, A., Shergaui, M.Y., Magid, S.E.A. and Abd-Alla, M.D. (2020) Harmonic Oscillator Solution for Free and Time Independent Potential String within the Frame Work of Dirac Special Relativistic Equation. IOSR Journal of Applied Physics, 12, 7-9.

10. Attia, I.A., Dirar, M. and Rasha, A. (2018) Quantization of Harmonic Oscillator Solution by Friction Term Method. International Journal of Theoretical and Mathematical Physics, 8, No. 4.

11. Ahmed Mohamed, E., Dirar, M., Hammad, I.A.I., Elfaki, A. and Alfaki, I. (2018) Potential Dependent Frictional Schrodinger Equation. American Scientific Research Journal for Engineering, Technology, and Sciences, 39, No. 1.

12. Ahmed Mohamed, E., Ali Ahmed, N., Babiker Hussein, M., Mohammad Taha, R., Ahmed, M. and Abd-Alla, M. (2020) Explanation of Pressure Effect for High Temperature Superconductors Using Pressure Dependent Schrodinger Equation and String Theory. Natural Science, 12, 28-34. https://doi.org/10.4236/ns.2020.121004 